\title{
WHAT ARE APPROPRIATE INITIAL AND SALVAGE THERAPIES FOR PATIENTS WITH THROMBOTIC THROMBOCYOPENIC PURPURA (TTP)?
}

\author{
Leo McCarthy ${ }^{1}$,Atillio Orazi $^{1}$, Charles Miraglia ${ }^{1}$, Daniel Waxman', Elaine Skipworth ${ }^{1}$, Constance Danielson ${ }^{1}$
}

Indiana University Medical Center, Indianapolis, IN, USA: Department of Pathology and Laboratory Medicine' ${ }^{1}$ Indiana Blood Center, Indianapolis, IN, USA: Department ${ }^{2}$

Summary: Although much has been learned about the pathophysiologic process of thrombotic thrombocytopenic purpura (TTP), both diagnostically and therapeutically, since its initial description by Moschowitz in 1924, its etiology and treatments remain, in many instances, problematic. Thrombotic thrombocytopenic purpura remains a rare entity whose etiology is usually unknown, but several drugs and infections have now been implicated in its development. Although treatment by plasma exchange has gained worldwide acceptance, the optimal exchange media is not known, nor the volume and duration of exchange therapy, not appropriate salvage therapies. Without the benefit of randomized controlled trials, its treatment, to a large extent, remains not evidence-based but "eminence-based", making the same mistakes with increasing confidence over decades.

Key words: Cryopoor plasma; Fresh frozen plasma; Metalloproteinase; Moschowitz; Plasma exchange Prednisone; Solvent detergent plasma; von Willebrand factor

\section{Introduction}

Much progress has been made in understanding the pathophysiology and treatment of TTP since it was first reported by Moschowitz in $1925(1,2,6,11,12)$. However, because of its relatively infrequent occurrence, $1-3 /$ million, no randomized controlled trials have been completed (15). Therefore, some aspects of its treatment(s) remain either unknown or uncertain. We treated our first patient in 1975 and have now treated 161 patients with TTP, perhaps the largest series yet reported.

We also present our experience in utilizing splenectomy as salvage therapy for 15 refractory patients who failed prolonged plasma exchange therapy as well as immunosuppression. Splenectomy was quite commonly used as therapy prior to 1977 when PE became accepted as the most efficacious therapy $(13,14)$. The literature contains few descriptions of splenectomy for this process since then and the histopathologic changes of TTP in the spleen are rarely, if ever, mentioned (5). This appears to be the largest series of such patients as well.

\section{Diagnosis}

The diagnosis of TTP remains, in many instances, problematic even today. The classical pentad of profound thrombocytopenia, microangiopathic hemolytic anemia, neurological dysfunction, typically fluctuating, mild renal dysfunction and fever is many times not present. Recently, some believe even, if only anemia and thrombocytopenia are present, the diagnosis should be made and PE therapy begun. This approach is problematic in that the differential diagnosis for anemia and thrombocytopenia is lengthy and includes many other entities, i.e., DIC, ITP, several microangiopathic anemias, collagen diseases, most notably, Lupus erythematosus (4), none of which benefit from plasma exchange therapy. Most of these disease states may, in fact, be aggravated by exchange therapy rather than beginning efficacious therapies.

\section{Plasma Exchange}

We begin PE therapy on an emergent basis, usually $<6 \mathrm{hr}$ after admission, using large dialysis catheters for central venous access (7). A one plasma volume exchange is done daily until the patient recovers. Virtually all patients receive $1 \mathrm{mg} /$ kilogram of prednisone and we do not provide platelets for transfusion even when central lines are placed (8). We have observed no clinical bleeding in any of these patients during their line placements even though the majority have low platelet counts, i.e. 20k or below. We initially used FFP as replacement media until it became apparent the uLvWf 
multimers (10) played a significant role in the pathogenesis of this process when we began using cryopoor plasma which lacks all or most of these multimers as does solvent detergent (SD) plasma. Also, these plasmas contain normal levels of specific metalloproteinase, which are in usually relatively low concentrations in these patients $(3,16)$. We used SD plasma exclusively for nearly four years. However, unfortunately, it has been removed from the U.S. market.

One hundred and twenty-eight patients of 161 are alive ( $80 \%)$, and 106 have been women $(66 \%)$. One hundred patients are alive without recurrences $(63 \%)$, and 28 patients remain alive with recurrences $(17 \%)$. Thirty-four patients were exchanged exclusively with solvent detergent plasma and $32(>90 \%)$ survived. Forty-eight patients received only cryopoor plasma as exchange media, 14 survived (60\%). Sixty-two patients received only FFP and 47 survived (76 \%). Sixteen patients received portions of all three plasmas, and 15 survived. The response and survival rate of nearly $90 \%$ of SD plasma is superior to the other plasmas used for exchanges. Also, of considerable importance is the near-complete elimination of allergic reactions of patients exchanged with SD plasma. Only three patients had 1-2 small hives. Virtually all patients exchanged with FFP or cryopoor plasma have multiple reactions, some associated with bronchospasm and hypotension causing the exchanges to be interrupted and prolonged while allergic symptoms were treated. We have not observed Transfusion Related Acute Lung Injury reactions in any of our patients.

\section{Splenectomy}

Although used quite frequently prior to the widespread advent of transfusion related acute lung injury, plasma exchange therapy in the late 1970 s, little has been written about splenectomy for these patients since Schwartz (14) reviewed 32 long-term survivors, 21 of who received high dose of steroids and underwent splenectomies. Little can be found about the splenic histopathologic findings in TTP.

Between 1982 and 1998, 15 of 73 patients failed to respond adequately to intensive PE therapy and underwent splenectomies. Their clinical response to splenectomy and their eventual outcome were analyzed. The spleens were carefully examined for thrombi and subendothelial deposits as well as for trilineage extramedullary hematopoiesis by morphology and immuno-histochemistry for Factor VIII: RAg, myeloperoxidase, and hemoglobin.

None of the patients died secondary to splenectomy. To the best of our knowledge, 9 patients are still alive and none have relapsed. Nine spleens were enlarged with mean weights of $213 \pm 70 \mathrm{~g}$ (median $193 \mathrm{~g}$; range 150 to $392 \mathrm{~g}$ ). Ten spleens displayed thrombi/subendothelial deposits and 11 spleens exhibited extramedullary hematopoiesis, primarily erythropoiesis (9).
Intrasplenic platelet destruction may have a significant role in the pathogenesis of TTP in some patients and splenectomy may, therefore, be beneficial to them. Definitive histopathologic changes in the majority of these spleens also supports the beneficial role of splenectomy for some refractory patients. Nonetheless, the role of splenectomy remains controversial as treatment for TTP. However, we believe it still should be strongly considered as a treatment option for extremely refractory patients who have clearly failed prolonged plasma exchange and appropriate immunosuppressive therapies.

\section{References}

1. Baehr G, Klemperer P, Schifrin A. An acute febrile anemia and thrombocytopenic purpura with diffuse platelet thrombosis of capillaries and arterioles. Trans Assoc Am Physicians 1936;51:43-58.

2. Bukowski R, Hewlett $\mathbf{J}$, Harris $\mathbf{J}$ et al. Exchange transfusions in the treatment of thrombotic thrombocytopenic purpura. Semin Hematol 1976;3:219-32.

3. Furlan M, Robles R, Galbusera M et al. von Willebrand factor-cleaving protease in thrombotic thrombocytopenic purpura and hemolytic uremia syndrome. N Engl J Med 1998;339:1578-84.

4. Jain R, Chartash M, Myron S et al. Systemic Lupus Erythematosus Complicated by Thrombotic Microangiopathy. Semin Arthritis Rheum December 1994; 24(3):173-182

5. Kass L, Schnitzer B. Extra medullary haematopoiesis in thombotic thrombocytopenic purpura. Folia Haematol. 1979;106:32-36.

6. McCarthy LJ, Danielson CFM, Skipworth EM, Peters SL, Miraglia CC, Antony AC. Myocardial infarction/Injury is Relatively Common at Presentation of Acute Thrombotic Thrombocytopenic Purpura: The Indiana University Experience. Ther Apher 2002;6(1):2-4

7. McCarthy LJ, Danielson CFM, Rothenberger SS. Indications for Emergency Apheresis Procedures. Crit Rev Clin Lab Sci 1997;34(6):573-610.

8. McCarthy LJ, Danielson CF, Graves V et al. Do platelet transfusions to patients with TTP influence their survival? Blood 1994;84(Suppl 1):669a.

9. McCarthy LJ, Orazi A, Danielson CFM et al. Should splenectomy be considered for patients with refractory thrombotic thrombocytopenic purpura? (abstract) $51^{\text {st }}$ Annual Meeting of the American Association of Blood Banks, Philadelphia, PA, October 31-November 4, 1998. Transfusion, Special Abstract Supplement, October 1998;38:7S.

10. Moake JL et al. Unusually large plasma factor VIII: von Willebrand factor multimers in chronic relapsing thrombotic thrombocytopenic purpura. N Engl J Med 1982;307:1432.

11. Moschcowitz E. Hyaline thrombosis of the terminal arterioles and capillaries: a hitherto undescribed disease. Proc NY Pathol Soc 1924;24:21-4.

12. Rock GA, Shumak KH, Buskard NA, et al: Comparison of plasma exchange with plasma infusion. N Engl J Med 1991;325:393-7.

13. Rodriguez HF, Babb DF, Santiago, EP, et al. Thrombotic thrombocytopenic purpura: Remission after splenectomy. N Engl J Med 1957;257:983.

14. Schwartz J, Rosenberg A, Cooperberg AA. Thrombotic thrombocytopenic purpura: Successful treatment of two cases. Can Med Assoc J 1972;106:1200.

15. Torok et al. Incidence Rates. Am J Hematol 1995;50-84.

16. Tsai HM, Lian ECY. Antibodies to von Willebrand factor-cleaving protease in acute thrombotic thrombocytopenic purpura. N Engl J Med 1998;339: 1585-94.

Submitted December 2003

Accepted December 2003.

Leo J. McCarthy, MD, FRCP Edin/Ire, Emeritus Professor of Pathology/Medicine/Pediatrics, Department of Pathology and Laboratory Medicine, Indiana University Hospital, Room 4552, 550 North University Boulevard, Indianapolis, Indiana 46202-5283. e-mail: Immcarth@iupui.edu 